\section{Recovery of Cryptococcus gattii from an Infected Ventriculo-Peritoneal Shunt, Illinois, USA}

\author{
Donna Moritz, Alfredo J. Mena Lora, \\ Bridget Blumer, Amanda T. Harrington
}

Author affiliations: University of Illinois at Chicago, Chicago, Illinois, USA (D. Moritz, A.J. Mena Lora); ACL Laboratories, Rosemont, Illinois, USA (B. Blumer); Loyola University Medical Center, Maywood, Illinois, USA (A.T. Harrington)

DOI: https://doi.org/10.3201/eid2407.171754

Cryptococcus gattii is a fungal pathogen endemic in tropical and subtropical regions. Isolated cases and outbreaks have been reported in areas of North America and Europe, expanding the distribution pattern beyond warmer regions. We describe a case of ventriculo-peritoneal shunt infection by $C$. gattii in an immunocompetent person in Illinois.

C ryptococcus gattii is a fungus found in soil and decaying organic materials (1-3). C gattii infections have been reported in tropical and subtropical regions worldwide. In the United States, C. gattii human infection is rare; $<300$ cases have been documented, of which 169 were reported to the Centers for Disease Control and Prevention (CDC) during 2005-2013 (4). Most cases were reported in southern California before a rise in cases occurred after 1999 in the Pacific Northwest $(2,5)$. We report a case of ventricular shunt infection by $C$. gattii in an immunocompetent person in Illinois.

A 40-year-old man from Lake County, Illinois, with no known medical problems was admitted in October 2015 for evaluation of hydrocephalus. The patient reported 4 months of throbbing frontal headaches, nausea, and vomiting. Progressive confusion, altered memory, intermittent gait, and balance disturbances also were present. No visual changes, fevers, chills, or seizures were reported. The patient had no travel outside of Illinois and no ill contacts. Computed tomography (CT) scan of the brain demonstrated hydrocephalus, which was concerning because it indicated possible abnormalities in the flow of cerebrospinal fluid (CSF). A right frontal ventriculostomy catheter was placed. Repeat CT imaging of the head showed a possible mass within the right cerebellar hemisphere and surrounding vasogenic edema. To determine whether an infectious pathogen was the cause, we performed a workup that included HIV screening, Echinococcus serologic testing, interferon gamma release assay, cysticercosis serologic testing, and 3 CSF cultures; all results were negative. Results of a complete blood count with differential and comprehensive metabolic panel were unremarkable. The patient was not receiving immunosuppressive therapy nor had any other known risk factors associated with immunosuppression. A right ventriculo-peritoneal (VP) shunt with a programmable valve was placed, and the patient was discharged to home in stable condition.

One month later, the patient was readmitted with recurring symptoms. CT imaging of the head showed stable ventricular size. Contrast-enhanced magnetic resonance (MR) of the brain showed abnormalities above the tentorium, possibly representing a cystic mass obstructing the foramen of Monroe bilaterally, with pronounced distention of both lateral ventricles. CSF studies showed a leukocyte count of $3 / \mu \mathrm{L}$ (reference range $0-5 / \mu \mathrm{L}$ ) with lymphocytic predominance (93\% [reference range $40 \%-80 \%$ ]) and protein level of $42 \mathrm{mg} / \mathrm{dL}$ (reference range $15-45 \mathrm{mg} / \mathrm{dL}$ ). We observed large round yeasts on Gram stain of CSF. The VP shunt was externalized. Cryptococcal antigen (Immy; Norman, OK, USA) was positive in the CSF (1:160 titer). Cultures from the CSF grew yeast that we identified as Cryptococcus neoformans by using matrix-assisted laser desorption/ionization time-of-flight mass spectrometry (Vitek MS IVD Database version 2; bioMérieux, Durham, NC, USA). We then subcultured the organism to CGB Agar (L-canavanine, glycine, bromothymol blue; Hardy Diagnostics, Santa Monica, CA) to differentiate C. neoformans from C. gattii. The organism, which produced blue coloration on CGB agar, was determined to be $C$. gattii and was confirmed as $C$. gattii molecular biotype VGI by multilocus sequence typing performed at CDC (Atlanta, Georgia, USA). Induction with amphotericin B and flucytosine was given for 14 days and high-dose fluconazole $(800 \mathrm{mg} / \mathrm{d})$ was subsequently given as consolidation therapy for 8 weeks. The dose was then decreased (to $200 \mathrm{mg} / \mathrm{d}$ ) for maintenance therapy. The patient was lost to follow-up after his first outpatient clinic visit.

We postulate that our patient likely had a cryptococcoma with a low organism burden on initial presentation. We found no cases of VP shunt infection attributable to $C$. gattii in the literature. Only 10 cases of VP shunt infections attributable to $C$. neoformans have been reported; the time from shunt placement to symptom onset ranged from 10 days to 20 years $(6,7)$. Six of 10 cases resulted from shunt placement in persons previously infected $(6,8)$. The patient we report had onset of symptoms 4 weeks after VP shunt placement, likely reflecting an underlying infection before VP shunt placement.

Only 4 isolates of $C$. gattii have been identified from the Midwest region of the United States; these isolates were identified as VGI and VGIII types (S. Lockhart, CDC, pers. comm., 2017 Jul 18) (9). A recent study demonstrated that a large subset of isolates from throughout the United States 
were VGI, including a cluster of isolates with a single multilocus sequence type originating in the southeastern United States (10). The isolate in this case was identified as molecular type VGI and by multilocus sequence typing was shown to have the same sequence type as isolates from patients in Florida and Georgia and isolates from the environment in Washington.

Infections attributable to $C$. gattii are not confined to tropical and subtropical regions. The case we describe serves to extend the known range of this organism to include Illinois. Infections might be missed, given that many laboratories do not routinely differentiate $C$. gattii from $C$. neoformans. Mortality rates can range from $13 \%$ to $33 \%$ (4). Thus, clinicians and laboratorians must have increased awareness of this emerging infectious disease.

\section{Acknowledgments}

We thank Shawn Lockhart and Colleen Lysen for help with the genotyping of the isolate.

\section{About the Author}

Dr. Moritz is an infectious disease physician for Franciscan Alliance Physician Network in northwest Indiana and a voluntary faculty member for the Indiana University School of Medicine. Her primary research interests include emerging infectious diseases and infection prevention and control.

\section{References}

1 Chen SC-A, Meyer W, Sorrell TC. Cryptococcus gattii infections. Clin Microbiol Rev. 2014;27:980-1024. http://dx.doi.org/10.1128/ CMR.00126-13

2. Espinel-Ingroff A, Kidd SE. Current trends in the prevalence of Cryptococcus gattii in the United States and Canada. Infect Drug Resist. 2015;8:89-97. http://dx.doi.org/10.2147/IDR.S57686

3. Hoang L, Philips P, Galanis E. Cryptococcus gattii: a review of the epidemiology, clinical presentation, diagnosis, and management of this endemic yeast in the Pacific Northwest. Clin Microbiol Newsl. 2011;33:187-95. http://dx.doi.org/10.1016/ j.clinmicnews.2011.11.003

4. CDC. C. gattii infection statistics [cited 2017 Oct 10]. https://www.cdc.gov/fungal/diseases/cryptococcosis-gattii/ statistics.html

5. Harris JR, Lockhart SR, Sondermeyer G, Vugia DJ, Crist MB, D'Angelo MT, et al. Cryptococcus gattii infections in multiple states outside the US Pacific Northwest. Emerg Infect Dis. 2013;19:1620-6. http://dx.doi.org/10.3201/eid1910.130441

6. Viereck MJ, Chalouhi N, Krieger DI, Judy KD. Cryptococcal ventriculoperitoneal shunt infection. J Clin Neurosci. 2014;21:2020-1. http://dx.doi.org/10.1016/j.jocn.2014.08.001

7. Ingram CW, Haywood HB III, Morris VM, Allen RL, Perfect JR. Cryptococcal ventricular-peritoneal shunt infection: clinical and epidemiological evaluation of two closely associated cases. Infect Control Hosp Epidemiol. 1993;14:719-22. http://dx.doi.org/10.2307/30148351

8. Mangham D, Gerding DN, Peterson LR, Sarosi GA. Fungal meningitis manifesting as hydrocephalus. Arch Intern Med. 1983; 143:728-31. http://dx.doi.org/10.1001/ archinte.1983.00350040118015

9. Lockhart SR, Iqbal N, Harris JR, Grossman NT, DeBess E, Wohrle R, et al. Cryptococcus gattii in the United States: genotypic diversity of human and veterinary isolates. PLoS One. 2013;8:e74737. http://dx.doi.org/10.1371/ journal.pone. 0074737

10. Lockhart SR, Roe CC, Engelthaler DM. Whole-genome analysis of Cryptococcus gattii, southeastern United States. Emerg Infect Dis. 2016;22:1098-101. http://dx.doi.org/10.3201/eid2206.151455

Address for correspondence: Amanda T. Harrington, Loyola University Medical Center, Clinical Microbiology Laboratory, Pathology, 2160 S. First Ave, Maywood, IL 60153, USA; email: amanda.harrington@lumc.edu

\section{Late or Lack of Vaccination Linked to Importation of Yellow Fever from Angola to China}

\section{Rui Song, ${ }^{1}$ Shengcan Guan, ${ }^{1}$ Shui Shan Lee, ${ }^{1}$ Zhihai Chen, Chen Chen, Lifen Han, Yanli Xu, Ang Li, Hui Zeng, Hanhui Ye, Fujie Zhang}

Author affiliations: Beijing Ditan Hospital, Capital Medical University, Beijing, China (R. Song, Z. Chen, Y. Xu, F. Zhang); Mengchao Hepatobiliary Hospital of Fujian Medical University, Fuzhou, China (S. Guan, L. Han, H. Ye); The Chinese University of Hong Kong, Shatin, Hong Kong, China (S.S. Lee); Beijing Key Laboratory of Emerging and Reemerging Infectious Diseases, Beijing (C. Chen, A. Li, H. Zeng)

DOI: https://doi.org/10.3201/eid2407.171868

During March and April 2016, 11 yellow fever cases were identified in China. We report epidemic and viral information for 10 of these patients, 6 of whom had been vaccinated before travel. Phylogenetic analyses suggest these viruses nested within the diversity of strains endemic to Angola, where an outbreak began in 2015.

$I^{\prime}$ December 2015, the first case of a major yellow fever outbreak was reported in Angola; the outbreak spread to Democratic Republic of the Congo (DRC) (http:// www.who.int/csr/don/13-april-2016-yellow-fever-angola/ en/). In addition to 965 confirmed cases in DRC during

${ }^{1}$ These authors contributed equally to this article. 